\title{
Astragaloside IV Enhances Melanogenesis via the AhR-Dependent AKT/GSK-3 $\beta / \beta$-Catenin Pathway in Normal Human Epidermal Melanocytes
}

\author{
Baoyi Liu $\mathbb{D}^{1,2}$ Yongyi Xie $\mathbb{D}^{1,2}$ and Zhouwei $\mathrm{Wu} \mathbb{D}^{2}$ \\ ${ }^{1}$ Department of Dermatology, Shanghai General Hospital, Shanghai Jiao Tong University School of Medicine, \\ Shanghai 200080, China \\ ${ }^{2}$ Department of Dermatology, Shanghai General Hospital, Shanghai Jiao Tong University, Shanghai 200080, China
}

Correspondence should be addressed to Zhouwei Wu; zhouwei.wu@shgh.cn

Received 13 September 2020; Revised 3 December 2020; Accepted 5 December 2020; Published 15 December 2020

Academic Editor: Francesca Mancianti

Copyright (c) 2020 Baoyi Liu et al. This is an open access article distributed under the Creative Commons Attribution License, which permits unrestricted use, distribution, and reproduction in any medium, provided the original work is properly cited.

Astragalus membranaceus root has been widely used for repigmentation treatment in vitiligo, but its mechanism is poorly understood. We sought to investigate the effect of astragaloside IV (AS-IV), a main active extract of the Astragalus membranaceus root, on melanin synthesis in normal human epidermal melanocytes (NHEMs) and to elucidate its underlying mechanisms. Melanin content, tyrosinase activity, qPCR, western blot, and immunofluorescence were employed. Specific inhibitors and small interfering RNA were used to investigate the possible pathway. AS-IV stimulated melanin synthesis and upregulated the expression of melanogenesis-related genes in a concentration-dependent manner in NHEMs. AS-IV could activate the aryl hydrocarbon receptor (AhR), and AS-IV-induced melanogenesis was inhibited in si-AhR-transfected NHEMs. In addition, we showed that AS-IV enhanced the phosphorylation of AKT and GSK-3 $\beta$ and nuclear translocation of $\beta$-catenin. AS-IV-induced MITF expression upregulation and melanin synthesis were decreased in the presence of $\beta$-catenin inhibitor FH353. Furthermore, AhR antagonist CH223191 inhibited the activation of AKT/GSK-3 $\beta / \beta$-catenin signaling, whereas the expression of CYP1A1 (marker of AhR activation) was not affected by the AKT inhibitor in AS-IV-exposed NHEMs. Our findings show that AS-IV induces melanogenesis through AhR-dependent AKT/GSK-3 $\beta / \beta$-catenin pathway activation and could be beneficial in the therapy for depigmented skin disorders.

\section{Background}

Vitiligo is a kind of depigmentation disease with about $0.1-2 \%$ prevalence in the world [1], characterized by patchy depigmentation of the skin due to the loss of functional epidermal melanocytes. Epidermal melanocytes are responsible for the melanin synthesis which is driven by three key enzymes: tyrosinase (TYR), tyrosinase-related protein-1 (TYRP-1), and TYRP-2 in mammals [2]. The expression and activity of these three enzymes are regulated by the microphthalmia-associated transcription factor (MITF) [2]. The expression of the MITF gene can be modulated by many transcriptional factors in response to various environmental stimuli [3].

Aryl hydrocarbon receptor (AhR) is a ligand-dependent transcription factor that resides in the cytoplasm of various types of cells, including normal human epidermal melanocytes (NHEMs) [4]. Upon binding to ligands, AhR can translocate into the nucleus and regulate the expression of AhR-targeted genes such as cytochrome P450 1A1 (CYP1A1) [5]. In addition, AhR can also control the expression of other genes by interacting with various signaling pathways or regulating the half-life of other transcription factors [5]. It is well known that $\mathrm{AhR}$ is an important environmental sensor involved in a number of biological processes, including immune responses, endocrine regulation, and some chemical metabolism [5]. Our previous studies showed that AhR activation played roles in immune dysregulation and inflammatory response in CD4+ T cells and keratinocytes [6, 7]. Recently, AhR has been reported to modulate pigment synthesis via interacting with 
melanogenic signaling [4], and dysregulated AhR pathway might be involved in the development of vitiligo [8].

Many kinds of plant-derived products, for example, 8methoxypsoralen (8-MOP), have been used for repigmentation treatment in vitiligo for thousands of years [9]. However, undesirable side effects of 8-MOP such as skin phototoxicity and risk of skin cancer limit its clinical applications $[10,11]$. Therefore, it is desirable to find new products with better therapeutic effect and less side effects. Astragalus membranaceus is widely used in herbal medicine formulas such as Shouwushengheitang, Qubaixiaobantang [12], and Yiqiqubai [13] which have been successfully applied to treat vitiligo. Lin et al. [14] observed that aqueous extracts of the Astragalus membranaceus root were able to stimulate melanocyte proliferation. Astragaloside IV (ASIV) is a main active component isolated from root of Astragalus membranaceus (Figure 1) [15]. Several studies have reported its pharmacological effects including antioxidation, anti-inflammation [15], antiscar [16], and antihair loss [17]. However, its effect on melanogenesis remains unknown.

Here, we aimed to evaluate the effect of AS-IV on melanogenesis in NHEMs and to explore the underlying mechanisms.

\section{Materials and Methods}

2.1. Cell Culture and Treatment. Primary human epidermal melanocytes (NHEMs) were isolated from human foreskin specimens obtained during circumcision surgery and cultured in Medium 254 (M254500) supplemented with HMGS-2 (S0163) and 1\% antibiotic-antimycotic (Gibco, Grand Island, NY, USA). This study was approved by the ethics committee of Shanghai General Hospital (2017KY005) and conducted according to the principles of the Declaration of Helsinki. Cells were cultured in a humidified atmosphere with $5 \% \mathrm{CO}_{2}$ at $37^{\circ} \mathrm{C}$. Second- and fourth-passage NHEMs were used in all experiments. AS-IV (1044108), 6-formylindolo [3,2-b]carbazole (FICZ, SML1489), 8-MOP (M3501), AKT inhibitor VI (124013), FH535 (F5682), and CH223191 (C8124) were purchased from Sigma-Aldrich (St. Louis, MO, USA) and were dissolved in DMSO.

2.2. Cell Viability Assay. After treatment for $48 \mathrm{~h}, 10 \%$ of CCK-8 reagent (Yeasen, Shanghai, China) was added to each well and incubated for further $4 \mathrm{~h}$ at $37^{\circ} \mathrm{C}$. The metabolic activation of CCK- 8 was quantified by measuring the absorbance at $450 \mathrm{~nm}$ by using the spectrophotometer (Thermo Fisher Scientific Oy, Vantaa, Finland).

2.3. Melanin Measurement and Tyrosinase Assay. NHEMs were treated with AS-IV at concentrations of $0,1,10$, and $100 \mu \mathrm{M}$ or 8 -MOP at $100 \mu \mathrm{M}$ for $24 \mathrm{~h}$. Cells were lysed and centrifuged to obtain supernatant for tyrosinase activity and precipitate for melanin content assays. Then, $30 \mu \mathrm{g}$ protein of the supernatant was mixed with $100 \mu \mathrm{l} 0.1 \%$ L-DOPA in PBS at $\mathrm{pH} 6.8$ and incubated at $37^{\circ} \mathrm{C}$ for $30 \mathrm{~min}$, and the tyrosinase activity was measured at $475 \mathrm{~nm}$. The precipitate was dissolved in $100 \mu \mathrm{l}$ of $1 \mathrm{M} \mathrm{NaOH}$ with $10 \%$ DMSO for $1 \mathrm{~h}$ at $80^{\circ} \mathrm{C}$ to obtain melanin solution. The melanin content of melanin solution was measured at $405 \mathrm{~nm}$.

2.4. Quantitative Polymerase Chain Reaction ( $q P C R$ ). Total RNA was extracted using RNAiso Plus (Takara, Kyoto, Japan) and was transcribed into cDNA using PrimeScript ${ }^{\mathrm{TM}}$ RT Master Mix (Takara, Kyoto, Japan). The relative expression of various target genes was determined by q-PCR using TB Green ${ }^{\circledR}$ Premix Ex Taq ${ }^{\mathrm{TM}}$ II (Takara, Kyoto, Japan). Gene expression was normalized to that of GAPDH. Primer sequences used in this study are listed in Supplementary Table S1.

2.5. Western Blot Analysis. After treatment, NHEMs were lysed with RIPA Lysis Buffer or Nuclear and Cytoplasmic Protein Extraction Kit (Beyotime, Shanghai, China). About $20 \mu \mathrm{g}$ protein was separated by $10 \%$ SDS-PAGE and transferred to PVDF membranes (Merck Millipore, Billerica, MA, USA). After blocked in 5\% bovine serum albumin (BSA, Yeasen, Shanghai, China) for $1 \mathrm{~h}$, the membranes were incubated with primary antibodies overnight at $4^{\circ} \mathrm{C}$ and with secondary antibodies for $1 \mathrm{~h}$. GAPDH and nup98 were used as the loading control. Bound antibodies were detected using the ECL western blotting detection system (Merck Millipore, Burlington, MA, USA). The information for primary antibodies used in the experiment is listed in Supplementary Table S2.

2.6. Transfection with AhR-Targeted Specific Small Interference RNA. AhR-siRNA (AM4611) and negative control siRNA were purchased from Ambion (Waltham, MA, USA). NHEMs were incubated with a mix of lipofectamine 3000 (Invitrogen, Carlsbad, CA, USA) and si-AhR (10 nM) or negative control siRNA for $24 \mathrm{~h}$. Then, the NHEMs were treated with or without AS-IV for another $24 \mathrm{~h}$.

2.7. Immunofluorescence. After treatment, NHEMs were washed once and fixed with $4 \%$ paraformaldehyde for 20 min, permeabilized with $0.3 \%$ Triton X-100 in PBS for $20 \mathrm{~min}$, and blocked with $1 \%$ BSA for $30 \mathrm{~min}$ at room temperature. Samples were incubated with the anti-AhR (1: 20) antibody at $4^{\circ} \mathrm{C}$ for $8 \mathrm{~h}$ and then incubated with the Alexa Fluor 488 secondary antibody $(1: 500$, Invitrogen, Carlsbad, CA, USA) for $1 \mathrm{~h}$ at room temperature. Nuclei were stained with DAPI. Finally, images were captured using Leica TCS SP8 X Confocal (Leica, Mannheim, Germany).

2.8. Statistical Analysis. All data were presented as means \pm SD and were analyzed using one-way ANOVA followed by Tukey's or Dunnett's multiple comparisons after the test. All analyses were performed using GraphPad Prism 7 software (San Diego, CA, USA). Differences were regarded as significant at $P<0.05$. 


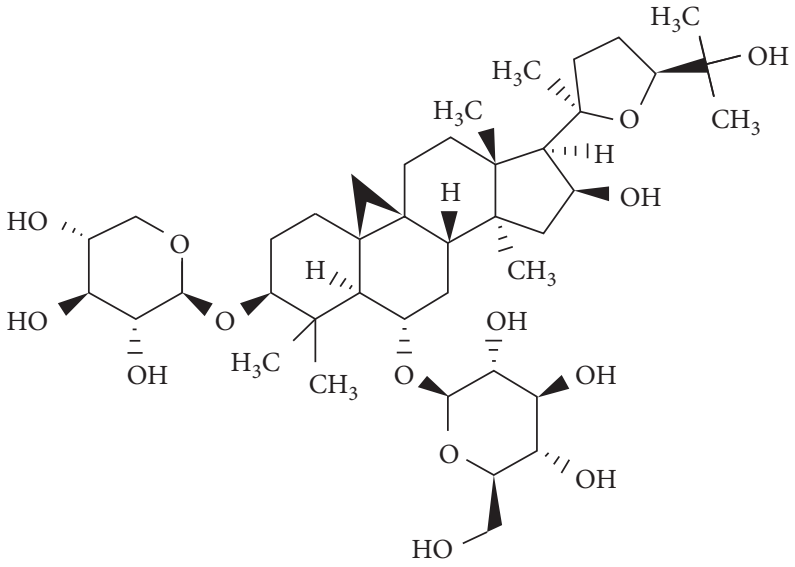

FIgUre 1: Chemical structure of AS-IV.

\section{Results}

3.1. AS-IV Stimulated Melanin Synthesis in a ConcentrationDependent Manner in NHEMs. First of all, we detected the cytotoxicity of AS-IV on NHEMs. NHEMs were incubated with AS-IV at concentrations ranging from 0 to $100 \mu \mathrm{M}$ for $48 \mathrm{~h}$, and then the viability of NHEMs was assessed using the CCK-8 cell viability assay. Our results showed that AS-IV had no cytotoxic effect on NHEMs and did not affect cell morphology (Figures 2(a) and 2(b)).

We then determined whether AS-IV could induce melanin synthesis in NHEMs. NHEMs were treated with various concentrations of AS-IV $(0,1,10$, and $100 \mu \mathrm{M})$ for $24 \mathrm{~h}$, and our results revealed that the melanin synthesis was increased by $15 \%, 35 \%$, and $72 \%$ respectively. In addition, we also showed that tyrosinase activity was increased by $10 \%$, $23 \%$, and $48 \%$, respectively. Furthermore, AS-IV at $100 \mu \mathrm{M}$ exhibited more effective in melanogenesis in NHEMs compared to $100 \mu \mathrm{M}$ 8-MOP (positive control, Figures 2(c) and $2(\mathrm{~d}))$.

3.2. AS-IV Upregulated the Expression of Melanogenesis-Related Genes in a Concentration-Dependent Manner in NHEMs. To elucidate the mechanisms underlying AS-IVmediated melanogenesis, we quantified the expression of TYR, TYRP-1, TYRP-2, and MITF. NHEMs were exposed to AS-IV $(0,1,10$, and $100 \mu \mathrm{M})$ for $24 \mathrm{~h}$, and the mRNA expression levels of TYR, TYRP-1, TYRP-2, and MITF were increased in a concentration-dependent manner. We also determined that the upregulation effect of AS-IV $(100 \mu \mathrm{M})$ on the expression of these genes was more robust than that caused by $100 \mu \mathrm{M}$ 8-MOP (Figure 3(a)). Likewise, western blot analysis demonstrated the protein expression levels of these genes were also upregulated in a concentration-dependent manner in NHEMs after $24 \mathrm{~h}$ treatment of AS-IV (Figures 3(b) and 3(c)).

3.3. AhR Signaling Was Essential for AS-IV-Induced Melanogenesis in NHEMs. Given that flavonoids are exogenous ligands of AhR [18], we reasoned that AS-IV might activate AhR in NHEMs. Therefore, we investigated whether AS-IV could lead to AhR nuclear translocation and increase the expression of AhR-targeted gene CYP1A1 in NHEMs. NHEMs were treated with AS-IV $(100 \mu \mathrm{M})$ or 6-formylindolo [3,2-b] carbazole (FICZ, $100 \mathrm{nM}$, positive control) for $4 \mathrm{~h}$. Immunofluorescence staining showed that AS-IV clearly induced the translocation of the AhR protein into the nuclei, while AhR was mainly localized in the cytoplasm in an unstimulated condition (Figure 4(a)). Consistently, the protein expression of AhR was increased in the nuclei, while it decreased in the cytoplasm after AS-IV treatment for $4 \mathrm{~h}$ (Figure 4(b)). Moreover, the mRNA and protein expression of CYP1A1 were upregulated by AS-IV in a concentrationdependent manner in NHEMs after $12 \mathrm{~h}$ treatment (Figures 4(c) and 4(d)). These data suggested that AS-IV could activate AhR in NHEMs.

To validate the essential role of AhR in AS-IV-induced melanogenesis, we transfected NHEMs with si-AhR and then exposed the cells to AS-IV at $100 \mu \mathrm{M}$ for $24 \mathrm{~h}$. As shown in Figure 4(e), the expression of AhR was successfully knocked down, and the increased MITF protein expression and melanin synthesis induced by AS-IV in the si-control condition were cancelled in the si-AhR condition (Figures 4(f) and 4(g)). These data indicated that AhR signaling was required for melanin synthesis induced by ASIV in NHEMs.

3.4. AKT/GSK-3 $/ \beta$-Catenin Pathway Was Involved in AS-IVInduced Melanogenesis in NHEMs. Since AKT/GSK-3 $\beta /$ $\beta$-catenin signaling plays a critical role in the melanogenesis [19], we investigated the effect of AS-IV on this pathway. As shown in Figures 5(a) and 5(b), AS-IV significantly enhanced AKT and GSK- $3 \beta$ phosphorylation and increased the $\beta$-catenin protein expression level in a concentration-dependent manner in NHEMs after $24 \mathrm{~h}$ treatment. We also showed that the nucleus-to-cytoplasm ratio of the $\beta$-catenin protein was increased in NHEMs after AS-IV $(100 \mu \mathrm{M})$ exposure (Figure 5(c)). Furthermore, NHEMs were pretreated with $\beta$-catenin inhibitor FH535 $(25 \mu \mathrm{M})$ for $3 \mathrm{~h}$ and 


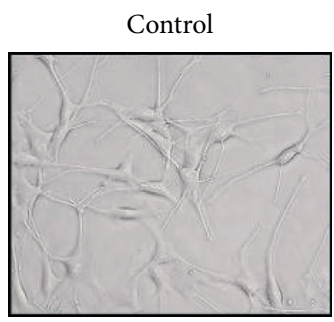

AS-IV $(100 \mu \mathrm{M})$

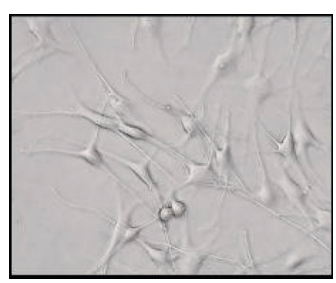

(a)

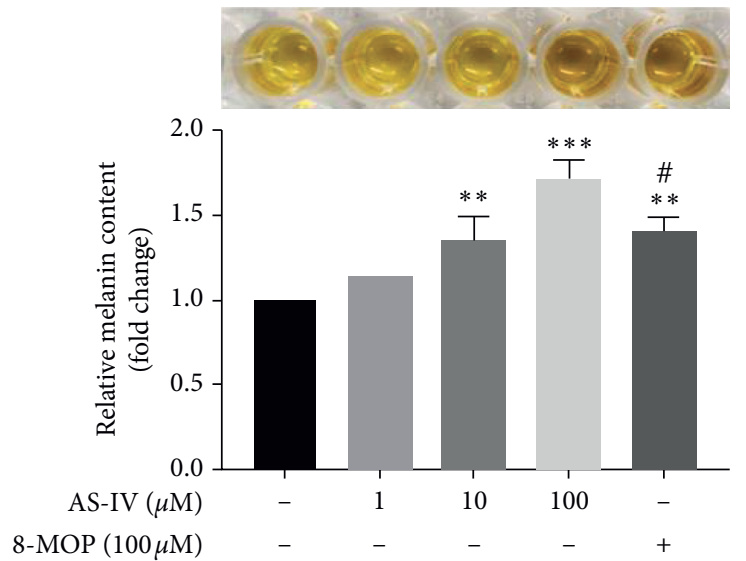

(c)

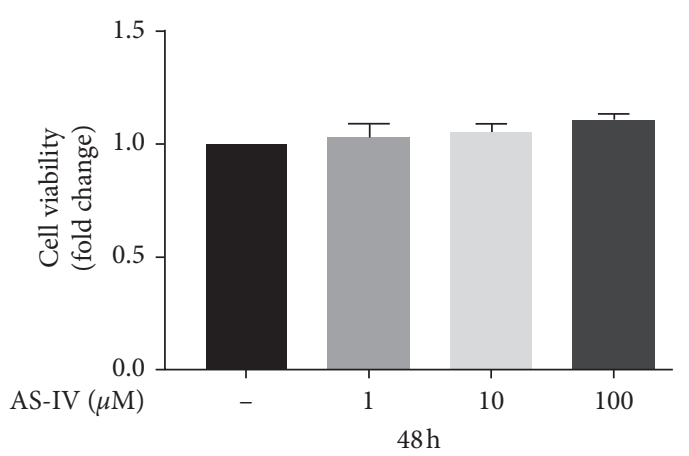

(b)

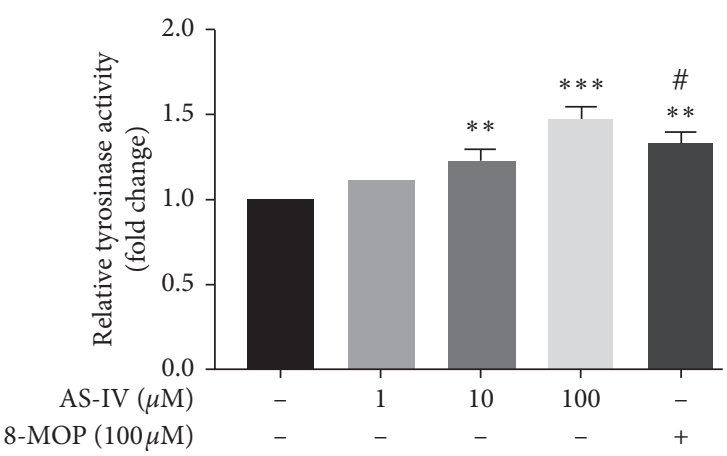

(d)

FIGURE 2: Effects of AS-IV on cell viability and melanogenesis in NHEMs. NHEMs were incubated with various concentrations $(0,1,10$, and $100 \mu \mathrm{M}$ ) of AS-IV for $48 \mathrm{~h}$ for (a) morphology (magnification: 40x) and (b) cell viability; NHEMs were incubated with various concentrations $(0,1,10$, and $100 \mu \mathrm{M})$ of AS-IV or 8 -MOP $(100 \mu \mathrm{M})$ for $24 \mathrm{~h}$ for $(\mathrm{c})$ melanin synthesis detection and (d) tyrosinase activity. Results were expressed as mean \pm SD $(n=3)$ based on the representative experiment of at least three replicates. ${ }^{* *} P<0.01$ and ${ }^{* * *} P<0.001$, versus untreated cells; ${ }^{\#} P<0.05$, versus AS-IV-treated $(100 \mu \mathrm{M})$ cells according to one-way ANOVA followed by Tukey's multiple comparisons.
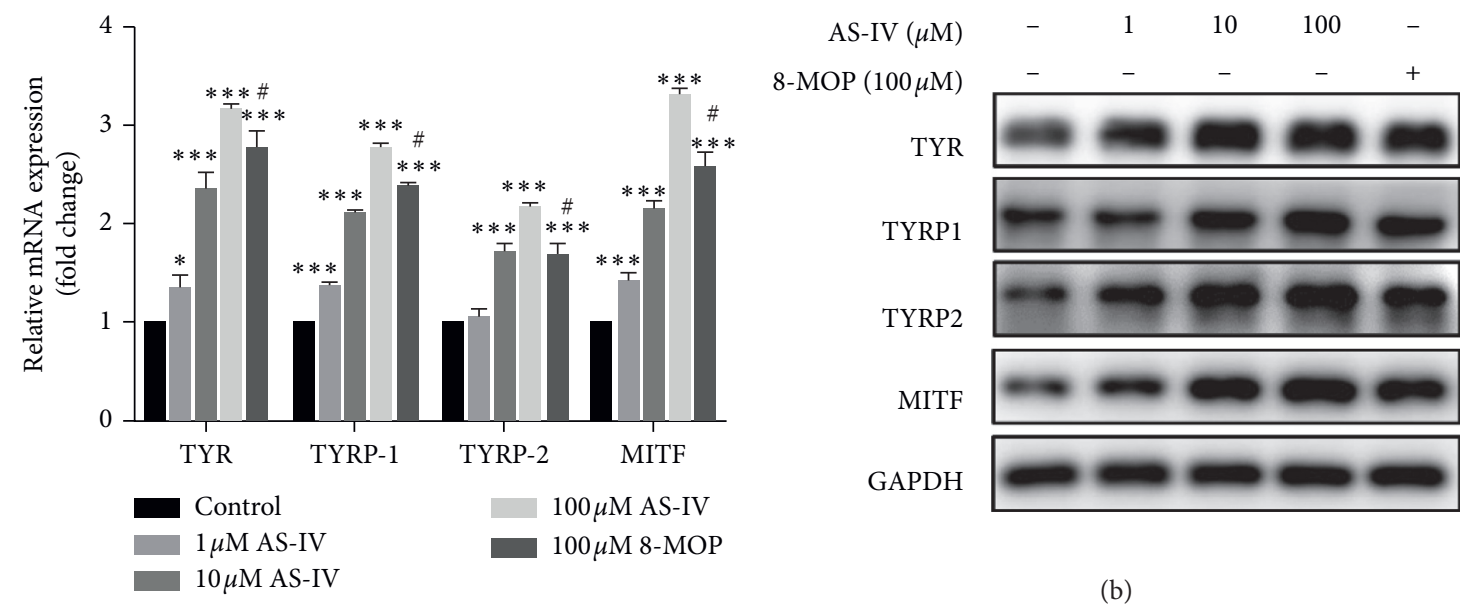

(a)

(b)

Figure 3: Continued. 


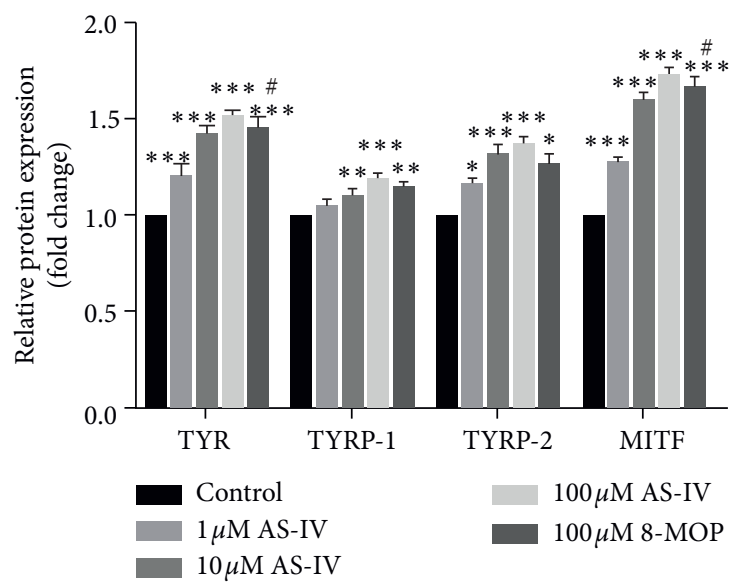

(c)

FIGURE 3: AS-IV upregulated the expression of melanogenesis-related genes in NHEMs. NHEMs were exposed to various concentrations (0, $1,10$, and $100 \mu \mathrm{M})$ of AS-IV or 8-MOP $(100 \mu \mathrm{M})$ for $24 \mathrm{~h}$ for (a) qPCR, (b) western blot, and (c) quantitative analyses. Results were expressed as mean $\pm \mathrm{SD}(n=3)$ of one representative experiment of at least three replicates. ${ }^{*} P<0.01,{ }^{* *} P<0.01$, and ${ }^{* * *} P<0.001$, versus untreated cells; ${ }^{\#} P<0.05$, versus AS-IV-treated $(100 \mu \mathrm{M})$ cells according to one-way ANOVA followed by Tukey's multiple comparisons.

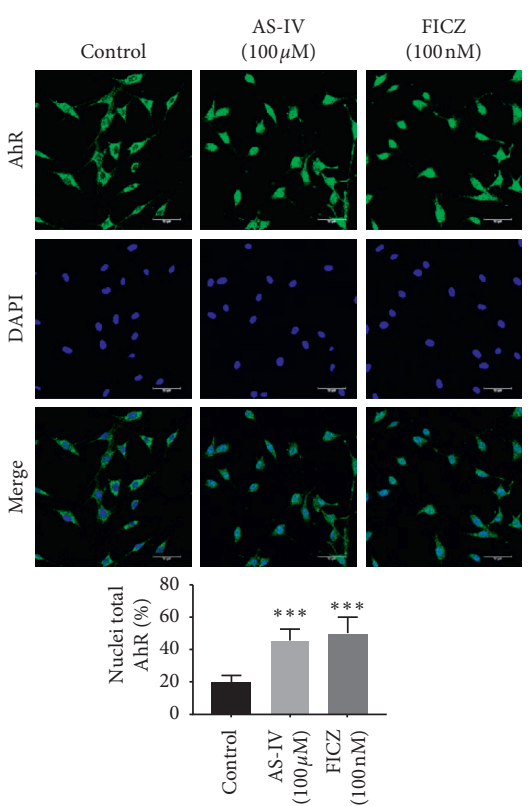

(a)

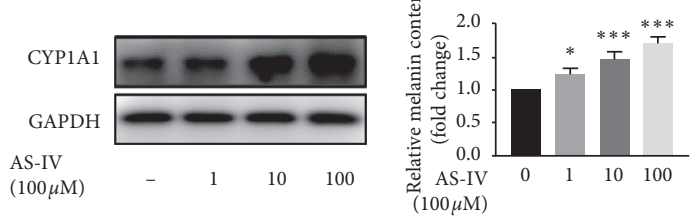

(d)
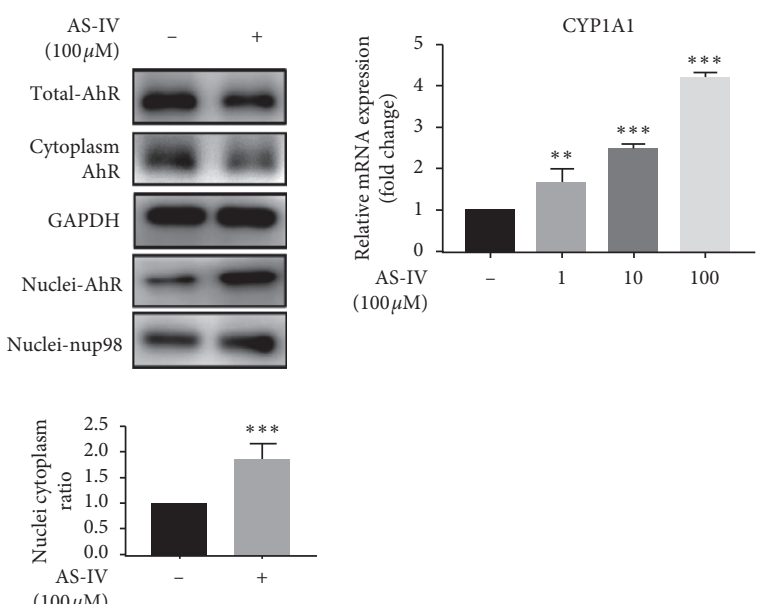

$\left(\begin{array}{cc}\mathrm{AS}-\mathrm{IV} \\ (100 \mu \mathrm{M})\end{array}\right.$

(b)

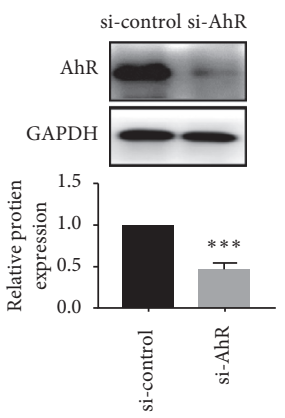

(e) (c)
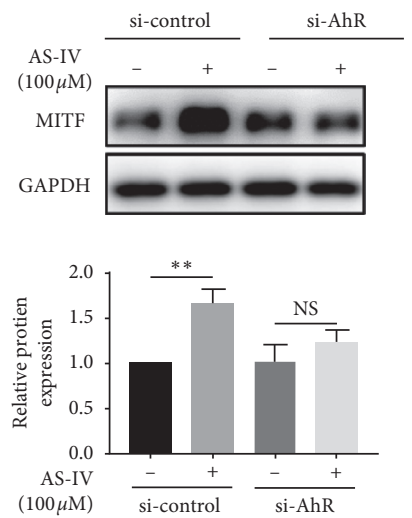

(f)

Figure 4: Continued. 


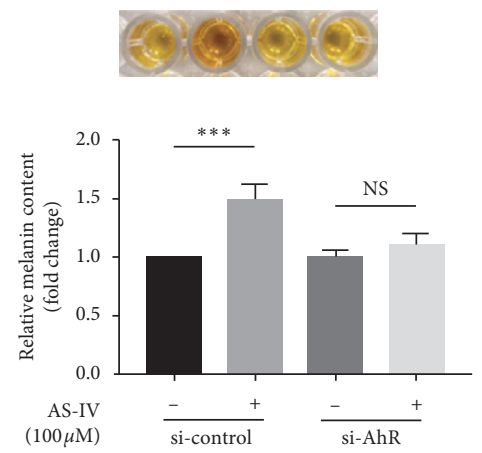

(g)

FIgURE 4: AhR signaling was essential for AS-IV-induced melanogenesis in NHEMs. NHEMs were incubated with AS-IV (100 $\mu \mathrm{M})$ or FICZ $(100 \mathrm{nM})$ for $4 \mathrm{~h}$. AhR nuclear translocation was detected by (a) confocal laser scanning microscopic analysis $(\mathrm{scale} b a \mathrm{r}=50 \mu \mathrm{m})$ and $(\mathrm{b})$ western blot analysis and quantitative analysis. NHEMs were incubated with various concentrations $(0,1,10$, and $100 \mu \mathrm{M})$ of AS-IV for $12 \mathrm{~h}$ or $24 \mathrm{~h}$ for (c) qPCR and (d) western blot and quantitative analyses. (e) The effect of si-AhR transfection. si-control and si-AhR-transfected NHEMs were treated with AS-IV at $100 \mu \mathrm{M}$ for $24 \mathrm{~h}$ for (f) western blot and quantitative analyses and (g) melanin content assay. Results were expressed as mean $\pm \mathrm{SD}(n=3)$, and images showed one representative experiment of at least three replicates. ${ }^{* *} P<0.01$ and ${ }^{* * *} P<0.001$ according to one-way ANOVA followed by Dunnett's multiple comparisons.

exposed to AS-IV $(100 \mu \mathrm{M})$ for $24 \mathrm{~h}$. We demonstrated that the AS-IV-stimulated MITF expression upregulation and melanin synthesis were almost cancelled in the presence of the $\beta$-catenin inhibitor (Figures $5(\mathrm{~d})$ and $5(\mathrm{e})$ ). These results supported the idea that the AKT/GSK-3 $\beta / \beta$-catenin pathway was involved in the AS-IV-induced melanogenesis in NHEMs.

3.5. AKT/GSK-3 $\beta / \beta$-Catenin Pathway Was Controlled by AhR Activation in Response to AS-IV in NHEMs. To explore the crosstalk between $\mathrm{AhR}$ and AKT/GSK-3 $\beta / \beta$-catenin signaling, NHEMs were pretreated with $\mathrm{CH} 223191$ (AhR inhibitor) or AKT inhibitor IV for $3 \mathrm{~h}$ and exposed to AS-IV $(100 \mu \mathrm{M})$ for $24 \mathrm{~h}$. We observed that AS-IV-induced AKT/ GSK-3 $\beta / \beta$-catenin signaling activation and MITF protein expression upregulation were reversed under both CH223191- and AKT inhibitor IV-pretreatment conditions in NHEMs. In addition, we found that the protein expression of CYP1A1 was decreased in the presence of CH223191 but not affected by AKT inhibitor IV (Figures 6(a) and 6(b)). Similarly, the AS-IV-stimulated melanin synthesis was reduced in both $\mathrm{CH} 223191$ - and AKT inhibitor IVpretreated conditions (Figure 6(c)).

\section{Discussion}

In the recent past, AhR has been regarded as a novel regulatory factor of human melanin synthesis [20]. $\mathrm{AhR}^{-/-}$mice showed lower tyrosinase activity [21], and mutations of the $A H R$ gene were also associated with vitiligo susceptibility
[22]. Consistently, lower transcription level of AhR was observed in the skin lesions of vitiligo patients [8]. These data imply that AhR may be a therapeutic target in depigmented skin diseases.

Many natural products can regulate melanogenesis through AhR activation [23]. AS-IV is a kind of natural flavonoids [24-26] which have been identified as exogenous ligands of AhR [27]. Currently, our study showed that AS-IV could stimulate the melanin synthesis via activating AhR in NHEMs.

It has been reported that AS-IV could exert its pharmacological activities through the AKT/GSK- $3 \beta / \beta$-catenin pathway $[28,29]$. In the present study, increased phosphorylation levels of AKT and GSK- $3 \beta$ were identified in ASIV-treated NHEMs. The phosphorylation of AKT increases GSK-3 $\beta$ phosphorylation, which further prevents $\beta$-catenin from degradation, increases its stability in the cytoplasm, and promotes its nuclear translocation $[19,30]$. In line with this notion, increased nucleus-to-cytoplasm ratio of the $\beta$-catenin protein was detected in AS-IV-treated NHEMs. $\beta$-Catenin can further upregulate MITF transcription by binding to the MITF promoter together with LEF1 in nuclei, increases the expression of TYR, TYRP-1, and TYRP-2 $[19,31]$, and ultimately promotes the melanin synthesis (Figure 7).

Recently, several studies have focused on the crosstalk between AhR and other signal pathways [32-35]. It has been reported that TCDD, a high-affinity ligand of AhR, was able to increase the phosphorylation level of AKT and GSK-3 $\beta$ [36] and activate $\beta$-catenin [34]. Our study showed that AS-IVinduced AKT/GSK-3 $\beta / \beta$-catenin pathway activation and 


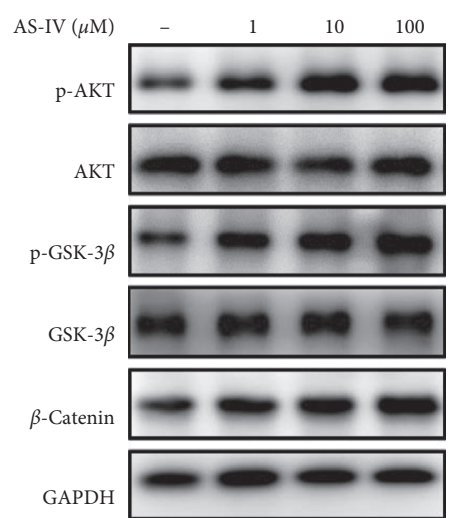

(a)

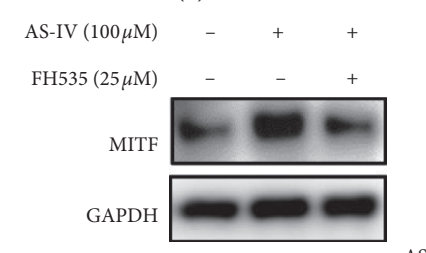

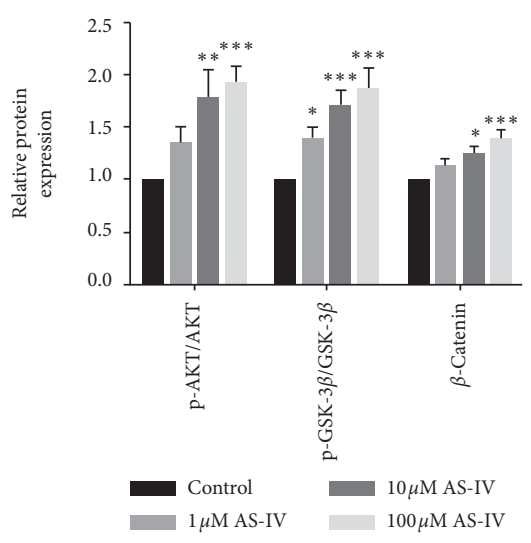

(b)

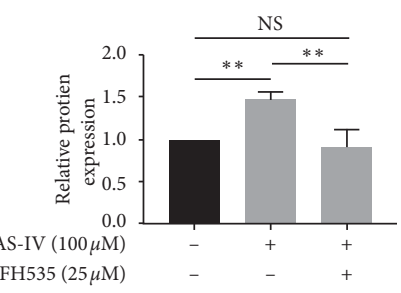

(d)

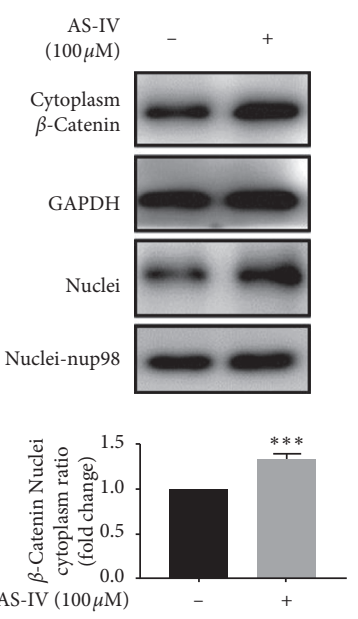

(c)

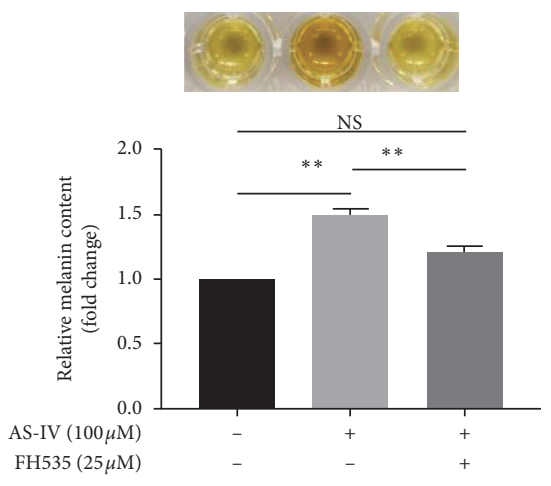

(e)

FIgUre 5: AKT/GSK-3 $\beta / \beta$-catenin pathway was involved in AS-IV-induced melanogenesis in NHEMs. NHEMs were incubated with various concentrations $(0,1,10$, and $100 \mu \mathrm{M})$ of AS-IV for $24 \mathrm{~h}$, and the protein expression of p-AKT, AKT, p-GSK-3 $\beta$, GSK-3 $\beta$, and $\beta$-catenin was analyzed by (a) western blot and (b) quantitative analyses. NHEMs were incubated with or without AS-IV (100 $\mu \mathrm{M})$ for $24 \mathrm{~h}$, and the protein expression of $\beta$-catenin in the cytoplasm and nucleus was analyzed by (c) western blot analysis and quantitative analysis. NHEMs were incubated with AS-IV $(100 \mu \mathrm{M})$ for $24 \mathrm{~h}$ with or without pretreatment with FH535 $(25 \mu \mathrm{M})$ for $3 \mathrm{~h}$. Protein expression of MITF was analyzed by (d) western blot and quantitative analyses, and (e) melanin synthesis was detected. Results were expressed as mean \pm SD $(n=3) .{ }^{* *} P<0.01,{ }^{* * *} P<0.001$, and NS indicates nonsignificance according to one-way ANOVA followed by Dunnett's multiple comparisons.

melanin synthesis were abrogated with the presence of $\mathrm{CH} 223191$, whereas the AKT inhibitor IV could only inhibit melanin synthesis but had no effect on the upregulated CYP1A1 expression caused by AS-IV. These data suggested that the AKT/GSK-3 $\beta / \beta$-catenin pathway might be the downstream signaling of AhR in response to AS-IV in NHEMs. The crosstalk between AhR and AKT/GSK-3 $\beta$ / $\beta$-catenin pathway is complicated and not fully understood. It has been reported that AhR could activate AKT in a ROSdependent manner [37] or through binding Src kinase in the cell membrane [38]. In addition, the possibility that AhR triggers downstream effectors to promote $\beta$-catenin nuclear translocation or binds with $\beta$-catenin to move together into the nuclei cannot be excluded. Thus, the exact mechanism needs further investigation. It has been reported that $T Y R$, TYRP-1, and TYRP-2 genes might have multiple AhRbinding sites in their promoter regions [20]. In our study, we also showed that melanin synthesis induced by AS-IV was partially inhibited in the presence of AKT inhibitor IV raising the question whether AhR could induce melanogenesis through other pathways rather than AKT/GSK-3 $\beta / \beta$-catenin signaling. This issue will be addressed in our future study. 


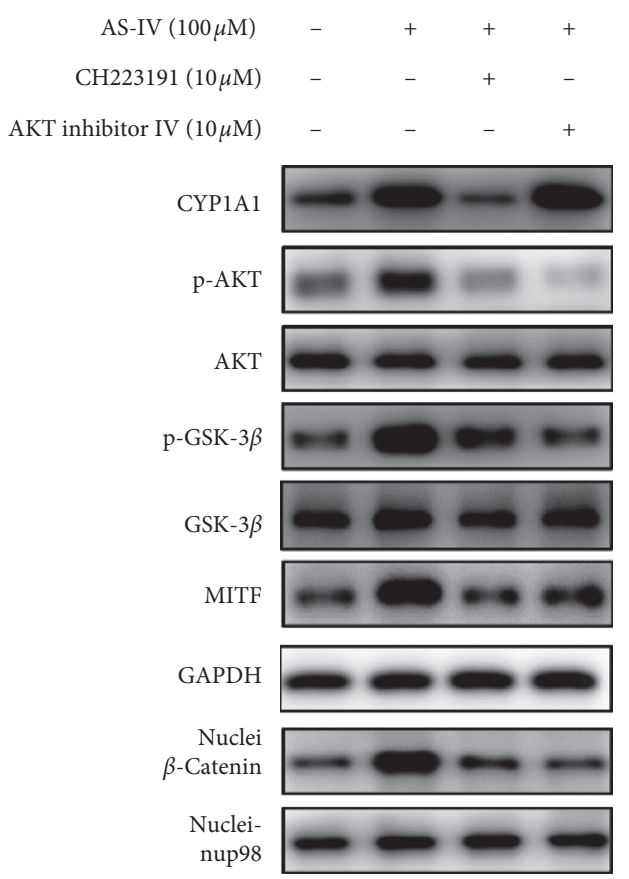

(a)

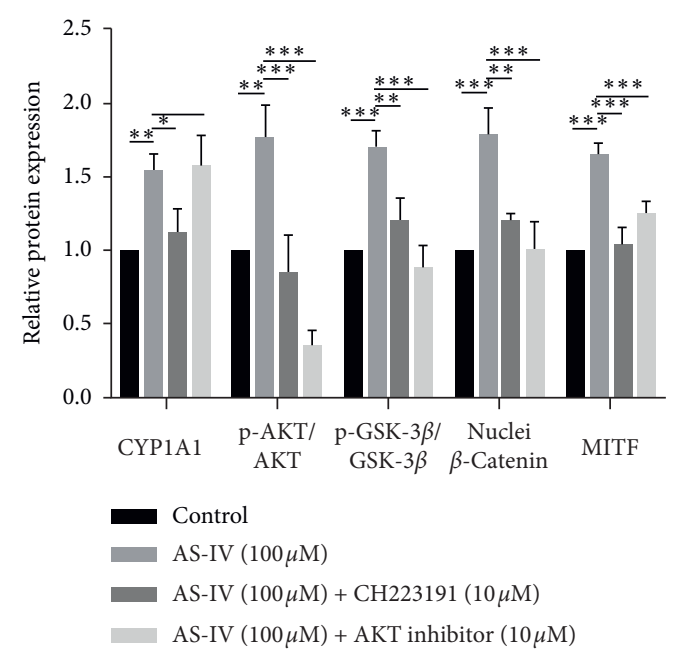

(b)

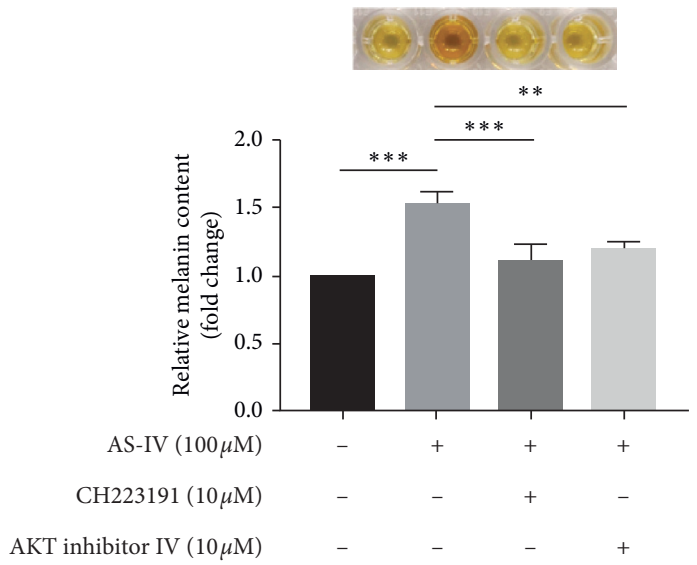

(c)

FIGURE 6: AKT/GSK-3 $\beta / \beta$-catenin pathway was controlled by AhR activation in response to AS-IV in NHEMs. NHEMs were incubated with AS-IV $(100 \mu \mathrm{M})$ for $24 \mathrm{~h}$ with or without pretreatment with AKT inhibitor IV $(10 \mu \mathrm{M})$ or CH223191 (10 $\mu \mathrm{M})$ for $3 \mathrm{~h}$. Protein expressions of CYP1A1, p-AKT, AKT, p-GSK-3 $\beta$, GSK- $3 \beta$, MITF, and nuclei $\beta$-catenin were analyzed using (a) western blot and (b) quantitative analyses; (c) melanin synthesis detection. Results were expressed as mean $\pm \mathrm{SD}(n=3) .{ }^{* *} P<0.01$ and ${ }^{* * *} P<0.001$ according to one-way ANOVA followed by Dunnett's multiple comparisons.

There are some limitations in our study. NHEMs were used in our study as an in vitro model system, and further investigations on light- and dark-pigmented melanocytes are needed to provide comprehensive data on the effect of AS-IV. Moreover, although AS-IV stimulated melanin synthesis in our in vitro study, whether it induces normal skin hyperpigmentation in vivo needs more studies.
In conclusion, our study shows that AS-IV stimulates melanin synthesis in cultured NHEMs. This induction of melanogenesis by AS-IV is at least partially mediated by AhR-dependent AKT/GSK-3 $\beta / \beta$-catenin signaling and the subsequent upregulated expression of MITF. Medications containing AS-IV might be beneficial in repigmentation for vitiligo and other acquired hypopigmentation diseases. 

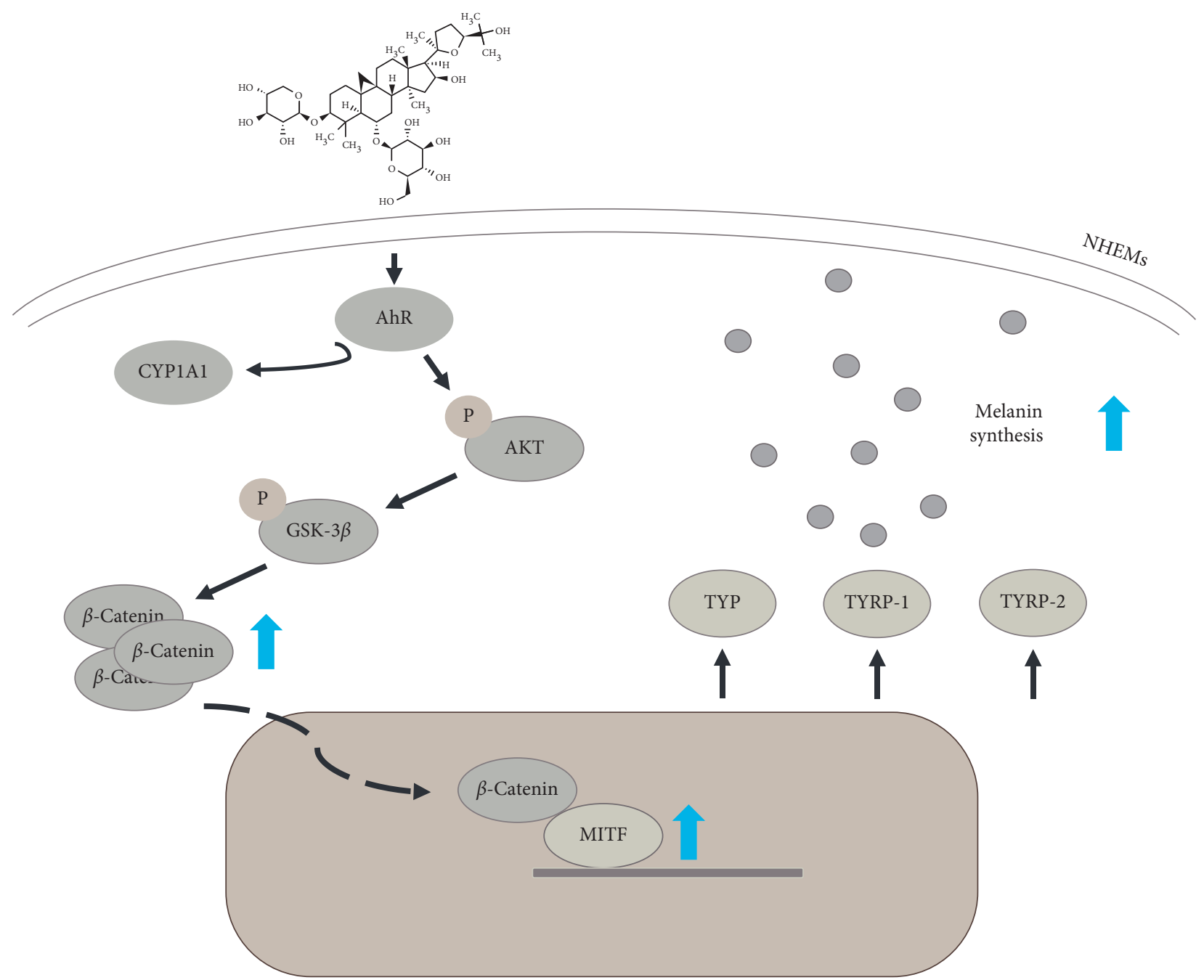

FIgURE 7: A proposed mechanism of AS-IV-induced melanogenesis. Astragaloside IV activates AhR, which then induces the phosphorylation of AKT and GSK-3 $\beta$ to promote $\beta$-catenin nuclear translocation. $\beta$-Catenin binds the promoter of the MITF gene to increase MITF transcription and subsequently upregulates TYR, TRP-1, and TRP-2 expressions and ultimately stimulates the melanin synthesis.

\section{Data Availability}

The primer sequence and antibody data used to support the findings of this study are included within the supplementary information file.

\section{Ethical Approval}

All skin samples were collected with the approval of the ethics committee of Shanghai General Hospital (2017KY005), Shanghai, China.

\section{Consent}

Written informed consent was obtained from all donors according to the Declaration of Helsinki.

\section{Conflicts of Interest}

The authors declare that they have no conflicts of interest.

\section{Authors' Contributions}

Zhouwei $\mathrm{Wu}$ and Baoyi Liu contributed to experimental design. Baoyi Liu and Yongyi Xie contributed to experimental operation. All authors agreed both to be personally accountable for their own contributions and to ensure that questions are related to the accuracy or integrity of any part of the work. All authors reviewed the manuscript and approved the submitted version. Baoyi Liu and Yongyi Xie contributed equally to this manuscript.

\section{Supplementary Materials}

Supplementary Table S1: primer sequences used for qPCR. Supplementary Table S2: antibodies used for western blot analysis. (Supplementary Materials)

\section{References}

[1] M. Rodrigues, K. Ezzedine, I. Hamzavi, A. G. Pandya, J. E. Harris, and G. Vitiligo Working, "New discoveries in the 
pathogenesis and classification of vitiligo," Journal of the American Academy of Dermatology, vol. 77, no. 1, pp. 1-13, 2017.

[2] S. H. Baek and S. H. Lee, "Sesamol decreases melanin biosynthesis in melanocyte cells and zebrafish: possible involvement of MITF via the intracellular cAMP and p38/JNK signalling pathways," Experimental Dermatology, vol. 24, no. 10, pp. 761-766, 2015.

[3] S. A. D’Mello, G. J. Finlay, B. C. Baguley, and M. E. AskarianAmiri, "Signaling pathways in melanogenesis," International Journal of Molecular Sciences, vol. 17, no. 7, Article ID 1144, 2016.

[4] M. Nakamura, Y. Ueda, M. Hayashi, H. Kato, T. Furuhashi, and A. Morita, "Tobacco smoke-induced skin pigmentation is mediated by the aryl hydrocarbon receptor," Experimental Dermatology, vol. 22, no. 8, pp. 556-558, 2013.

[5] C. Esser and A. Rannug, "The aryl hydrocarbon receptor in barrier organ physiology, immunology, and toxicology," Pharmacological Reviews, vol. 67, no. 2, pp. 259-279, 2015.

[6] Z. Wu, X. Mei, Z. Ying, Y. Sun, J. Song, and W. Shi, "Ultraviolet B inhibition of DNMT1 activity via AhR activation dependent SIRT1 suppression in CD4+ T cells from systemic lupus erythematosus patients," Journal of Dermatological Science, vol. 86, no. 3, pp. 230-237, 2017.

[7] Z. Wu, H. Uchi, S. Morino-Koga et al., "Z-Ligustilide inhibits benzo(a)pyrene-induced CYP1A1 upregulation in cultured human keratinocytes via ROS-dependent Nrf2 activation," Experimental Dermatology, vol. 23, no. 4, pp. 260-265, 2014.

[8] R. Rekik, A. Ben Hmid, C. Lajnef, I. Zamali, I. Zaraa, and M. Ben Ahmed, "Aryl hydrocarbon receptor (AhR) transcription is decreased in skin of vitiligo patients," International Journal of Dermatology, vol. 56, no. 12, pp. 1509-1512, 2017.

[9] Y. Li, J. Huang, J. Lu et al., "The role and mechanism of Asian medicinal plants in treating skin pigmentary disorders," Journal of Ethnopharmacology, vol. 245, Article ID 112173, 2019.

[10] S. Tippisetty, D. Goudi, A. W. Mohammed, and P. Jahan, "Repair efficiency and PUVA therapeutic response variation in patients with vitiligo," Toxicology in Vitro, vol. 27, no. 1, pp. 438-440, 2013.

[11] L. M. Felsten, A. Alikhan, and V. Petronic-Rosic, "Vitiligo: a comprehensive overview part II: treatment options and approach to treatment," Journal of the American Academy of Dermatology, vol. 65, no. 3, pp. 493-514, 2011.

[12] Y.-J. Chen, Y.-Y. Chen, C.-Y. Wu, and C.-C. Chi, "Oral Chinese herbal medicine in combination with phototherapy for vitiligo: a systematic review and meta-analysis of randomized controlled trials," Complementary Therapies in Medicine, vol. 26, pp. 21-27, 2016.

[13] C. Zhang, L. Zhou, J. Huang, and W. Shi, "A combination of yiqiqubai granule and $308 \mathrm{~nm}$ excimer laser in treatment of segmental vitiligo: a prospective study of 233 patients," Journal of Dermatological Treatment, vol. 28, no. 7, pp. 668-671, 2017.

[14] Z. X. Lin, J. R. S. Hoult, and A. Raman, "Sulphorhodamine B assay for measuring proliferation of a pigmented melanocyte cell line and its application to the evaluation of crude drugs used in the treatment of vitiligo," Journal of Ethnopharmacology, vol. 66, no. 2, pp. 141-150, 1999.

[15] J. Zhu and K. Wen, "Astragaloside IV inhibits TGF- $\beta 1$-induced epithelial-mesenchymal transition through inhibition of the PI3K/Akt/NF- $\kappa$ B pathway in gastric cancer cells," Phytotherapy Research, vol. 32, no. 7, pp. 1289-1296, 2018.
[16] X. Chen, L. H. Peng, N. Li et al., "The healing and anti-scar effects of astragaloside IV on the wound repair in vitro and in vivo," Journal of Ethnopharmacology, vol. 139, no. 3, pp. 721-727, 2011.

[17] M. H. Kim, S.-H. Kim, and W. M. Yang, "Beneficial effects of astragaloside IV for hair loss via inhibition of Fas/Fas L-mediated apoptotic signaling," PLoS One, vol. 9, no. 3, Article ID e92984, 2014.

[18] T. Yang, Y.-L. Feng, L. Chen, N. D. Vaziri, and Y.-Y. Zhao, "Dietary natural flavonoids treating cancer by targeting aryl hydrocarbon receptor," Critical Reviews in Toxicology, vol. 49, no. 5, pp. 445-460, 2019.

[19] D. Zang, C. Niu, and H. A. Aisa, "Amine derivatives of furocoumarin induce melanogenesis by activating Akt/GSK3beta/beta-catenin signal pathway," Drug Design, Development and Therapy, vol. 13, pp. 623-632, 2019.

[20] S. Luecke, M. Backlund, B. Jux, C. Esser, J. Krutmann, and A. Rannug, "The aryl hydrocarbon receptor (AHR), a novel regulator of human melanogenesis," Pigment Cell \& Melanoma Research, vol. 23, no. 6, pp. 828-833, 2010.

[21] B. Jux, S. Kadow, S. Luecke, A. Rannug, J. Krutmann, and C. Esser, "The aryl hydrocarbon receptor mediates UVB radiation-induced skin tanning," Journal of Investigative Dermatology, vol. 131, no. 1, pp. 203-210, 2011.

[22] X. W. Wang, K. Li, S. Guo et al., "The association of functional polymorphisms in the aryl hydrocarbon receptor (AHR) gene with the risk of vitiligo in Han Chinese populations," British Journal of Dermatology, vol. 166, no. 5, pp. 1081-1087, 2012.

[23] S. Fiorito, F. Epifano, F. Preziuso et al., "Natural oxyprenylated coumarins are modulators of melanogenesis," European Journal of Medicinal Chemistry, vol. 152, pp. 274-282, 2018.

[24] O. Zanoaga, C. Braicu, A. Jurj, A. Rusu, R. Buiga, and I. Berindan-Neagoe, "Progress in research on the role of flavonoids in lung cancer," International Journal of Molecular Sciences, vol. 20, no. 17, Article ID 4291, 2017.

[25] Z. Cai, J. Liu, H. Bian, and J. Cai, "Astragaloside IV ameliorates necrotizing enterocolitis by attenuating oxidative stress and suppressing inflammation via the vitamin D3-upregulated protein $1 / \mathrm{NF}-\kappa \mathrm{B}$ signaling pathway," $E x$ perimental and Therapeutic Medicine, vol. 12, no. 4, pp. 2702-2708, 2016.

[26] M. Ali, I.-D. Kim, S. Bilal et al., "Effects of bacterial fermentation on the biochemical constituents and antioxidant potential of fermented and unfermented soybeans using probiotic Bacillus subtilis (KCTC 13241)," Molecules, vol. 22, no. 12, Article ID 2200, 2017.

[27] C. Esser, A. Rannug, and B. Stockinger, "The aryl hydrocarbon receptor in immunity," Trends in Immunology, vol. 30, no. 9, pp. 447-454, 2016.

[28] D. Wei, H. Xu, X. Gai, and Y. Jiang, “Astragaloside IV alleviates myocardial ischemia-reperfusion injury in rats through regulating PI3K/AKT/GSK-3 $\beta$ signaling pathways," Acta Cirurgica Brasileira, vol. 34, no. 7, Article ID e201900708, 2019.

[29] L. Jia, D. Lv, S. Zhang, Z. Wang, and B. Zhou, “Astragaloside IV inhibits the progression of non-small cell lung cancer through the Akt/GSK-3 $\beta / \beta$-catenin pathway," Oncology Research Featuring Preclinical and Clinical Cancer Therapeutics, vol. 27, no. 4, pp. 503-508, 2019.

[30] P.-Y. Zhu, W.-H. Yin, M.-R. Wang, Y.-Y. Dang, and X.-Y. Ye, "Andrographolide suppresses melanin synthesis through Akt/ GSK3 $\beta / \beta$-catenin signal pathway," Journal of Dermatological Science, vol. 79, no. 1, pp. 74-83, 2015. 
[31] T. Pillaiyar, M. Manickam, and S.-H. Jung, "Downregulation of melanogenesis: drug discovery and therapeutic options," Drug Discovery Today, vol. 22, no. 2, pp. 282-298, 2017.

[32] K. W. Bock, "Aryl hydrocarbon receptor (AHR): from selected human target genes and crosstalk with transcription factors to multiple AHR functions," Biochemical Pharmacology, vol. 168, pp. 65-70, 2019.

[33] M. Murai, K. Yamamura, A. Hashimoto-Hachiya, G. Tsuji, M. Furue, and C. Mitoma, "Tryptophan photo-product FICZ upregulates AHR/MEK/ERK-mediated MMP1 expression: implications in anti-fibrotic phototherapy," Journal of Dermatological Science, vol. 91, no. 1, pp. 97-103, 2018.

[34] A. Al-Dhfyan, A. Alhoshani, and H. M. Korashy, "Aryl hydrocarbon receptor/cytochrome P450 1A1 pathway mediates breast cancer stem cells expansion through PTEN inhibition and $\beta$-catenin and Akt activation," Molecular Cancer, vol. 16, no. 1, p. 14, 2017.

[35] X. Liu, X. Li, Y. Tao et al., "TCDD inhibited the osteogenic differentiation of human fetal palatal mesenchymal cells through AhR and BMP-2/TGF- $\beta /$ Smad signaling," Toxicology, vol. 431, Article ID 152353, 2020.

[36] G. Xu, Y. Li, K. Yoshimoto et al., "2,3,7,8-tetrachlorodibenzop-dioxin stimulates proliferation of HAPI microglia by affecting the Akt/GSK-3 $\beta /$ cyclin D1 signaling pathway," Toxicology Letters, vol. 224, no. 3, pp. 362-370, 2014.

[37] T.-L. Liao, S.-C. Chen, C.-R. Tzeng, and S.-H. Kao, “TCDD induces the hypoxia-inducible factor (HIF)- $1 \alpha$ regulatory pathway in human trophoblastic JAR cells," International Journal of Molecular Sciences, vol. 15, no. 10, pp. 17733-17750, 2014.

[38] M. Ye, Y. Zhang, H. Gao et al., "Activation of the aryl hydrocarbon receptor leads to resistance to EGFR TKIs in nonsmall cell lung cancer by activating src-mediated bypass signaling," Clinical Cancer Research, vol. 24, no. 5, pp. 1227-1239, 2018. 